\title{
Saccharomyces boulardii improves the behaviour and emotions of spastic cerebral palsy rats through the gut-brain axis pathway
}

Deshuang Tao ${ }^{1,2 \dagger}$, Tangwu Zhong ${ }^{1 \dagger}$, Wei Pang ${ }^{3,4,5}$ and Xiaojie $\mathrm{li}^{3,4,5^{*}}$

\begin{abstract}
Background: Cerebral palsy (CP) is a kind of disability that influences motion, and children with CP also exhibit depression-like behaviour. Inflammation has been recognized as a contributor to CP and depression, and some studies suggest that the gut-brain axis may be a contributing factor. Our team observed that Saccharomyces boulardii (S. boulardii) could reduce the inflammatory level of rats with hyperbilirubinemia and improve abnormal behaviour. Both $\mathrm{CP}$ and depression are related to inflammation, and probiotics can improve depression by reducing inflammation. Therefore, we hypothesize that S. boulardii may improve the behaviour and emotions of spastic CP rats through the gut-brain axis pathway.

Methods: Our new rat model was produced by resecting the cortex and subcortical white matter. Seventeen-dayold CP rats were exposed to $S$. boulardii or vehicle control by gastric gavage for 9 days, and different behavioural domains and general conditions were tested. Inflammation was assessed by measuring the inflammatory markers IL-6 and TNF-a. Hypothalamic-pituitary-adrenal (HPA) axis activity was assessed by measuring adrenocorticotropic hormone and corticosterone in the serum. Changes in the gut microbiome were detected by 16S rRNA.
\end{abstract}

Results: The hemiplegic spastic CP rats we made with typical spastic paralysis exhibited depression-like behaviour. S. boulardii treatment of hemiplegic spastic CP rats improves behaviour and general conditions and significantly reduces the level of inflammation, decreases HPA axis activity, and increases gut microbiota diversity.

Conclusions: The model developed in this study mimics a hemiplegic spastic cerebral palsy. Damage to the cortex and subcortical white matter of 17-day-old Sprague-Dawley (SD) rats led to spastic CP-like behaviour, and the rats exhibited symptoms of depression-like behaviour. Our results indicate that $\mathrm{S}$. boulardii might have potential in treating hemiplegic spastic CP rat models or as an add-on therapy via the gut-brain axis pathway.

Keywords: Hemiplegic spastic cerebral palsy, Animal model, Saccharomyces boulardii, Gut microbiome, HPA axis, Inflammation, Depression-like behaviour, Immune regulation, Gut-brain axis, probiotics

\section{Introduction}

Cerebral palsy (CP) is a kind of disorder that influences motion and, in more severe forms, the disability of the patient to sit and stand independently [1]. CP

\footnotetext{
*Correspondence: dazhumama@yeah.net

†Deshuang Tao and Tangwu Zhong contributed equally to this work

${ }^{3}$ College of Rehab Medicine, Jiamusi University, Jiamusi, China

Full list of author information is available at the end of the article
}

is considered the most common cause of physical disability in childhood; In a majority of cases, the predominant motor abnormality is spasticity; other forms of $\mathrm{CP}$ include dyskinetic (dystonia or choreoathetosis) and ataxic $\mathrm{CP}$ [2]. The overall prevalence remains stable at 2-3.5 cases per 1000 live births [3]; approximately 1 in 345 children in the United States have been identified with CP, affecting more than 500,000 children and 
families in North America [4, 5]. Children with CP may develop a series of secondary symptoms over time. For example, children with $\mathrm{CP}$ have more psychological difficulties than normal children $[6,7]$. Fatigue, pain, and depressive symptoms are also common in adults with $\mathrm{CP}$ [8-10]. Depression-like behaviour in children and adults with $\mathrm{CP}$ is much more common than previously thought and is either not recognized or poorly managed by clinicians [11]. Except in the mildest cases, $\mathrm{CP}$ has a substantial impact on families' well being and societal health care costs [12].

The treatment of CP is mainly physical therapy [13, 14]. There are few studies on the treatment of spastic $C P$ depression-like behaviour. Probiotics can reduce proinflammatory cytokine levels, increase anti-inflammatory cytokine levels, and play an immune-regulatory role [15-17]. Saccharomyces boulardii (S. boulardii) is a kind of recognized probiotic [18]. S. boulardii could provide nutrients for the host, improve the activity of beneficial intestinal bacteria, inhibit the growth of pathogens, improve the immune function of the intestinal mucosa and then improve the various functions of the body [1921]. Our team observed that $S$. boulardii could reduce the inflammatory level of rats with hyperbilirubinemia, improve abnormal behaviour, and we propose the prospect of probiotics in the treatment of hyperbilirubinemia. Regarding spastic CP, to our knowledge, no study has investigated emotional status using probiotic treatment.

The term "gut-brain axis" refers to the bidirectional communication between the gut and the brain. The relationship between the gut-brain axis and the central nervous system (CNS) was determined to be mainly through the gut microbiota, immune system, and neuroendocrine (HPA axis) pathway [17]. Inflammation has been increasingly recognized as an important contributor to CNS disorders such as CP and depression [22-26]. Maternal immune activation (MIA) has been demonstrated to associate with $\mathrm{CP}$ in progeny [27]. Recent studies suggested that MIA disrupted inhibitory interneuron networks and that the gut-brain axis might be a contributing factor $[24,28,29]$. Probiotics could potentially improve depression-like behaviour by reducing inflammation. Gut microbiome manipulation in the form of probiotics is an effective therapeutic to ameliorate TBI-induced pathology and symptoms [30]. Prevention of gut leakiness by probiotic treatment leads to an attenuated HPA response to acute psychological stress in rats [15]. We hypothesized that $\mathrm{S}$. boulardii helped improve the behavioural and emotional symptoms of spastic $\mathrm{CP}$ through the gutbrain axis pathway.

Animal models are suitable platforms for conducting such studies [31]. Spastic CP correlated with injury of the cortex and subcortical white matter [32-34]. Many well-known and widely used animal models of spastic CP either show typical spastic CP-like phenotypes or overlook depression-like behaviour $[35,36]$. We endeavoured to establish a standardized hemiplegic spastic CPlike phenotype rat model that was similar to the clinical characteristics of human hemiplegic spastic CP. Based on the hemiplegic spastic CP-like phenotype rat model, the effects of probiotics on hemiplegic spastic CP rats could be observed, and the potential mechanism could be determined. The treatment of $\mathrm{CP}$ often ignores emotional problems. If $\mathrm{S}$. boulardii is widely used to alleviate emotional disorders in children with $\mathrm{CP}$, it will have great social significance.

\section{Results}

Hemiplegic Spastic CP rats had typical spastic CP-like phenotypes and depression-like behaviour

Referring to the atlas of the neonatal rat brain (Fig. 1A1, B1), compared with the control groups (Fig. 1-A2, $\mathrm{B} 2$ ), we destroyed the left-brain motor cortex and cingulate cortex of the $\mathrm{CP}$ groups by resecting the cortex and subcortical white matter. (Fig. 1-A3, B3). Rats in the CP groups showed typical paralysis (Fig. 2A) and high muscle tension (Fig. 2B). Longa and Ashworth scores were the highest. Three months later, the right hind paws of $\mathrm{CP}$ group rats were flexed, and the gaits of the right lower limbs were abnormal during walking (Fig. 2C). We measured the lower (Fig. 2D) and upper (Fig. 2F) limb muscle strengths, and there were significant differences between the CP groups and the control groups on day $9(\mathrm{p}<0.05)$. We also measured the neurological deficits, adductor angle, and muscle tension between the $\mathrm{CP}$ groups and the control groups on days 1-9, and there were significant differences between the control groups and the CP groups. Based on MRI scan findings and standardized neuromotor assessment, hemiplegic spastic CP rats can be diagnosed.

\section{S. boulardii ameliorated depression-like behaviour of hemiplegic spastic CP rats}

Regarding the evaluation of depression-like behaviour, we chose the tail suspension test (TST) (Fig. 2E) and sucrose preference test (SPT). The details of the results are shown in Table 8. The SPT showed a significant difference among the 3 groups $(\mathrm{F}=32.939, \mathrm{p}<0.01)$; the lower the score was, the more severe the depression. There were no significant differences in SPT scores in the control groups and $\mathrm{CP}+\mathrm{Sb}$ groups, while there was a significant increase in the $\mathrm{CP}+\mathrm{Sb}$ groups compared with the CP groups $(\mathrm{p}<0.01)$. The TST also showed a significant difference among the 3 groups $(F=187.439$, $\mathrm{p}<0.01)$ details: the higher the score was, the more severe the depression. The TST scores of the CP group were 


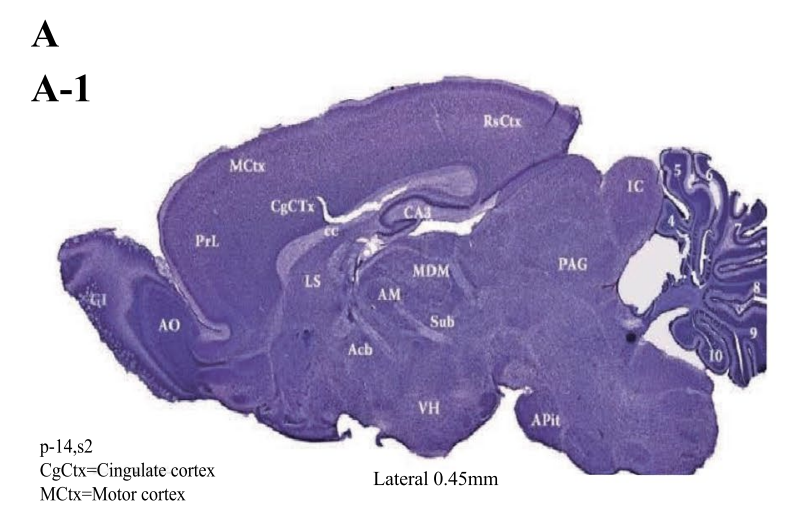

Atlas of NEONATAL RAT BRAIN (edit by Renuka Ramachandra and Thyagarajan Subramanian)

A-2

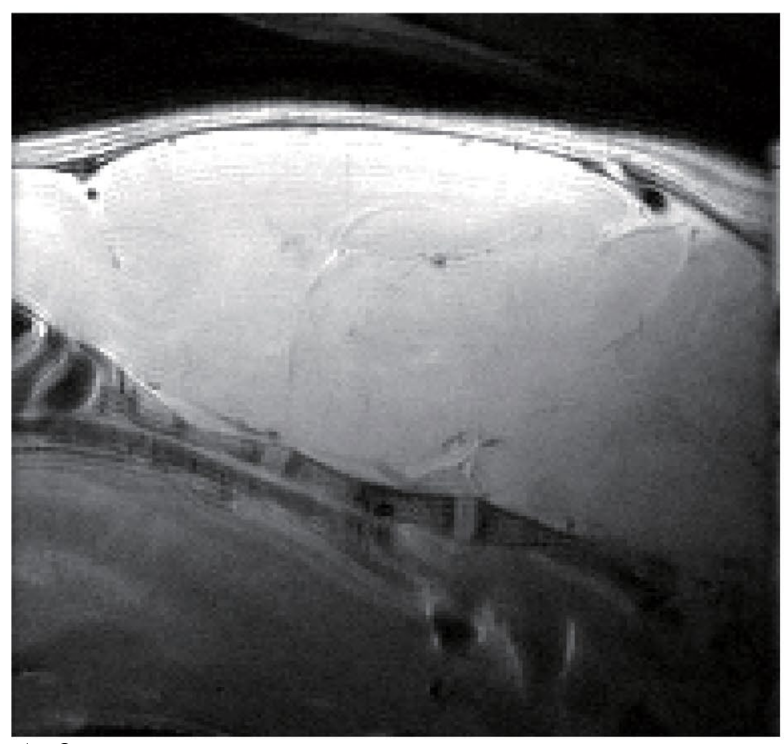

A-3

B

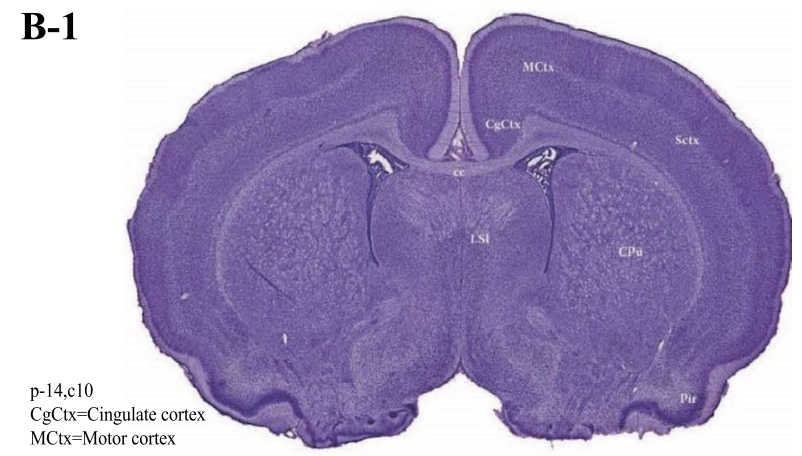

Atlas of NEONATAL RAT BRAIN (edit by Renuka Ramachandra and Thyagarajan Subramanian)

B-2
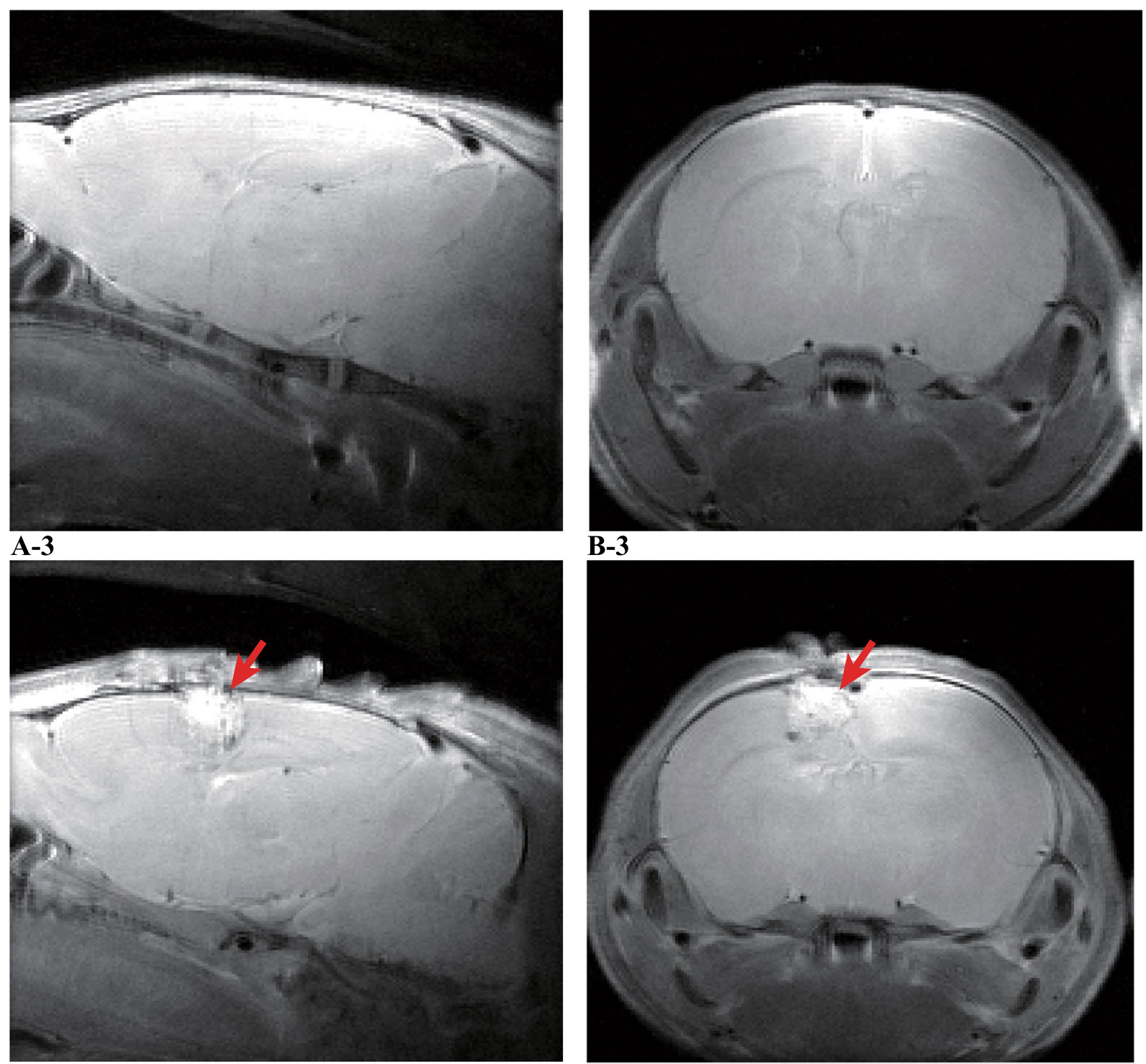

B-3

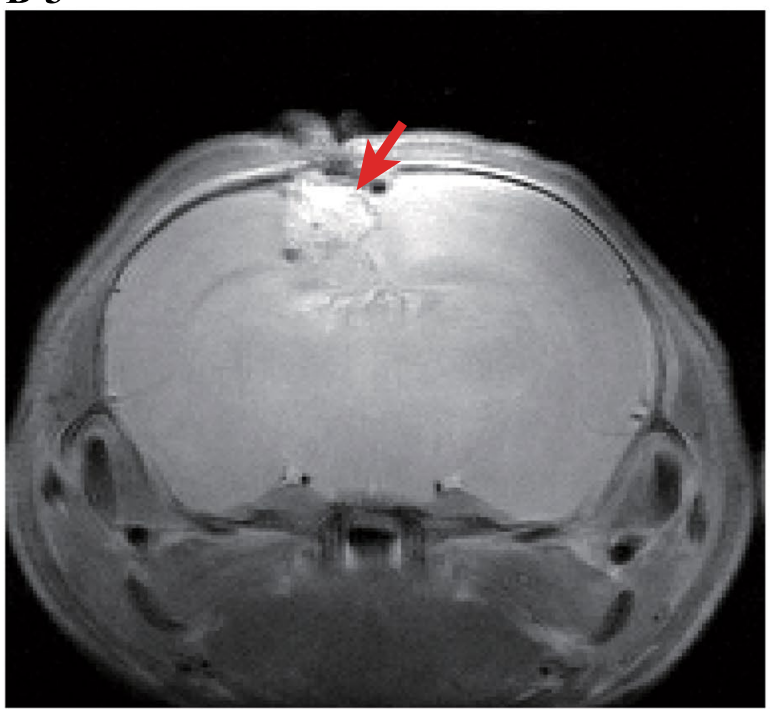

Fig. $1 \mathrm{MRI}$ results of the spastic cerebral palsy rat model. A-1 the sagittal view of the rat brain atlas. A-2 MRI results of normal rats. A-3 MRI results of model rats. B-1 the coronal view of the rat brain atlas. B-2 MRI results of normal rats. B-3 MRI results of model rats. The position indicated by the arrow is the surgical site 

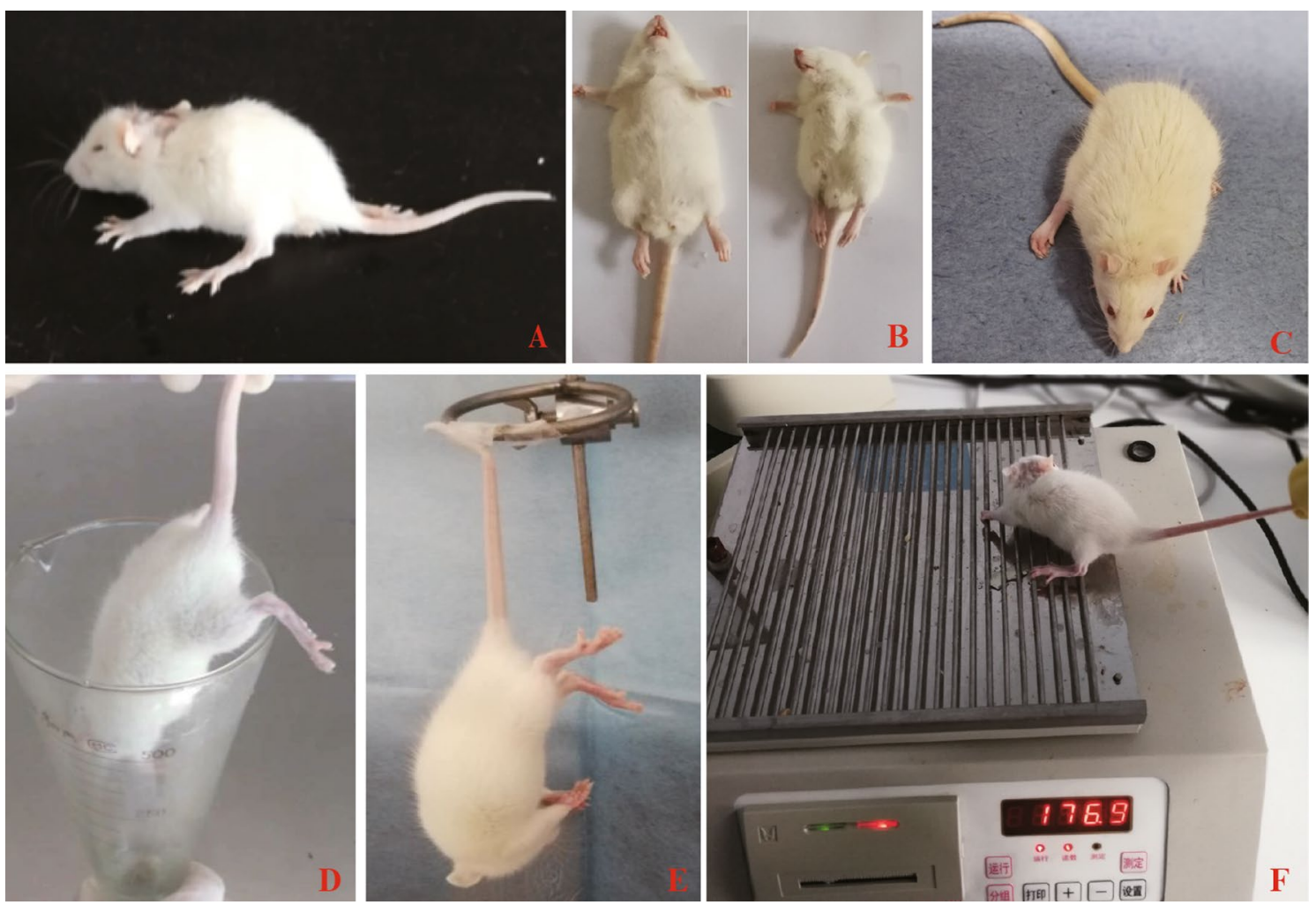

Fig. 2 Typical manifestations of postoperative rats. A The right lower limb of the rat is paralyzed. B The weight of the model group was lower than the weight of the normal group, the lower limb muscle tension was high, and the adductor angle was small. C After 3 months, the right lower paw of the rat was flexed, the muscle strength was weak, and the gait of the right lower limb was wider than the gait of the other side. D Hind limb suspension test. E. Tail suspension test. F. Grasping test

Table 1 Two-factor repeated-measures ANOVA of weight

\begin{tabular}{lrrrll}
\hline & \multicolumn{1}{l}{ SS } & df & \multicolumn{1}{l}{ MS } & F & p \\
\hline Group & 3485.013 & 2 & 1742.507 & 65.355 & 0.000 \\
Error (group) & 559.908 & 21 & 26.662 & & \\
Time & 3107.553 & 9 & 345.284 & 54.112 & 0.000 \\
Group * time & 1527.964 & 18 & 84.887 & 13.303 & 0.000 \\
Error (time) & 1205.998 & 189 & 6.381 & & \\
\hline
\end{tabular}

ANOVA analysis of variance, $S S$ stdev square, $d f$ degree of freedom, $M S$ mean square

*Interaction between time and group in the ANOVA

significantly higher than the TST scores of the $\mathrm{CP}+\mathrm{Sb}$ group ( $<<0.01)$, and the TST scores of the $\mathrm{CP}+\mathrm{Sb}$ group were significantly higher than the TST scores of the control group $(\mathrm{p}<0.01)$.

\section{S. boulardii ameliorated hemiplegic spastic CP rat weight, faecal water content and general state}

The results of hemiplegic spastic $\mathrm{CP}$ rat weights are shown in Table 1, Fig. 3A, and Additional file 3: Tables
S1 and S2. On Day 9, the control groups were significantly higher than $\mathrm{CP}+\mathrm{Sb}$ groups $(\mathrm{p}<0.01)$, and the $\mathrm{CP}+\mathrm{Sb}$ groups were significantly higher than $\mathrm{CP}$ groups $(p<0.01)$. The faecal water content results are shown in Table 2, Fig. 3B, and Additional file 3: Tables S3 and S4. On Day 9, the control groups were significantly lower than CP groups $(\mathrm{p}<0.01)$ while there were no significant differences between $\mathrm{CP}+\mathrm{Sb}$ groups and the control groups $(\mathrm{p}>0.05)$, and the $\mathrm{CP}+\mathrm{Sb}$ groups were significantly lower than $\mathrm{CP}$ groups $(\mathrm{p}<0.01)$. The general state scores are shown in Table 3, Fig. 3C, and Additional file 3: Tables S5 and S6. On Day 9, the control groups were significantly lower than the other 2 groups; the $\mathrm{CP}+\mathrm{Sb}$ groups were significantly lower than the $\mathrm{CP}$ groups $(\mathrm{p}<0.01)$.

\section{S. boulardii ameliorated neuromotor behaviour of hemiplegic spastic CP rats}

The results of neurological deficits are shown in Table 4, Fig. 4A, and Additional file 3: Tables S7 and S8. On Day 9, there were no significant differences between the $\mathrm{CP}+\mathrm{Sb}$ groups and the CP groups $(\mathrm{p}>0.05)$. The muscle tension 

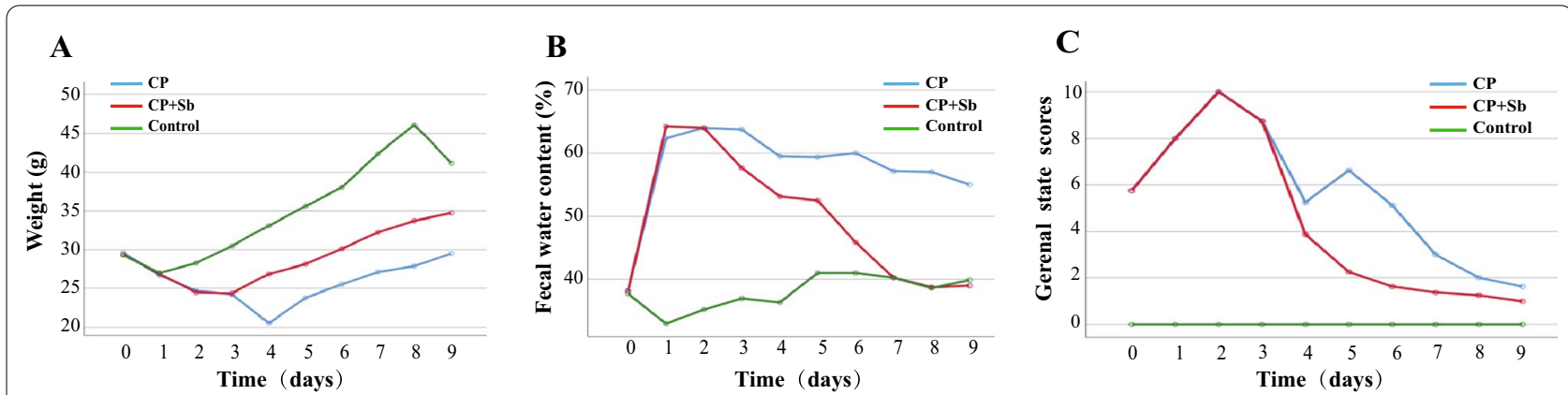

Fig. 3 Effect of Saccharomyces boulardii on spastic CP rats. A Daily changes in weight. B Daily changes of faecal water content. C Daily changes of general state score. $n=6$ in each group

Table 2 Two-factor repeated-measures ANOVA of faecal water content

\begin{tabular}{llrlll}
\hline & SS & df & MS & F & p \\
\hline Group & $15,529.658$ & 2 & 7764.829 & 476.126 \\
Error (group) & 342.475 & 21 & 16.308 & 0.000 \\
Time & 5471.167 & 9 & 607.907 & 75.327 & 0.000 \\
Group * time & 6758.758 & 18 & 375.487 & 46.527 & 0.000 \\
Error (time) & 1525.275 & 189 & 8.070 & \\
\hline
\end{tabular}

ANOVA analysis of variance, $S S$ stdev square, $d f$ degree of freedom, $M S$ mean square

*Interaction between time and group in the ANOVA

Table 3 Two-factor repeated-measures ANOVA of general state scores

\begin{tabular}{lrrrrr}
\hline & \multicolumn{1}{l}{ SS } & df & \multicolumn{1}{c}{ MS } & \multicolumn{1}{l}{$\mathbf{F}$} & \multicolumn{1}{c}{$\mathbf{p}$} \\
\hline Group & 1393.358 & 2 & 696.679 & 2225.135 & .000 \\
Error (group) & 6.575 & 21 & 9.313 & & \\
Time & 903.167 & 9 & 100.352 & 818.403 & .000 \\
Group * time & 539.058 & 18 & 29.948 & 244.234 & .000 \\
Error (time) & 23.175 & 189 & 9.123 & & \\
\hline
\end{tabular}

ANOVA analysis of variance, $S S$ stdev square, $d f$ degree of freedom, MS mean square

*Interaction between time and group in the ANOVA

Table 4 Two-factor repeated-measures ANOVA of neurological deficits

\begin{tabular}{lrrrrl}
\hline & \multicolumn{1}{c}{ SS } & df & \multicolumn{1}{c}{ MS } & F & p \\
\hline Group & 479.058 & 2 & 239.529 & $21,179.421$ & 0.000 \\
Error (group) & 0.238 & 21 & 0.011 & & \\
Time & 59.537 & 9 & 6.615 & 584.930 & 0.000 \\
Group * time & 34.025 & 18 & 1.890 & 167.140 & 0.000 \\
Error (time) & 2.138 & 189 & 0.011 & & \\
\hline
\end{tabular}

ANOVA analysis of variance, $S S$ stdev square, $d f$ degree of freedom, MS mean square

*Interaction between time and group in the ANOVA results are shown in Table 5 and Fig. $4 \mathrm{~B}$ and Additional file 3: Tables S9 and S10. The higher the scores, the more severe the neurological deficits. On Day 9, the control groups had significantly lower scores than the $\mathrm{CP}+\mathrm{Sb}$ groups $(\mathrm{p}<0.01)$, and the $\mathrm{CP}+\mathrm{Sb}$ groups had significantly lower scores than $\mathrm{CP}$ groups $(\mathrm{p}<0.01)$. The adductor angle results are shown in Table 6 and Fig. 4C and Additional file 3: Tables S11 and S12. The lower the scores were, the more severe the muscle tension. On Day 9 , the control groups had significantly higher scores than $\mathrm{CP}+\mathrm{Sb}$ groups $(\mathrm{p}<0.01)$, and the $\mathrm{CP}+\mathrm{Sb}$ groups had significantly higher scores than the CP groups $(\mathrm{p}<0.01)$. The grasping power results are shown in Table 7 and Fig. 4D and Additional file 3: Tables S13 and S14. The lower the scores were, the lower the muscle powers. On Day 9, the control groups had significantly higher scores than the $\mathrm{CP}+\mathrm{Sb}$ groups $(\mathrm{p}<0.01)$, and the $\mathrm{CP}+\mathrm{Sb}$ groups had significantly higher scores than the $\mathrm{CP}$ groups $(\mathrm{p}<0.01)$. The hind limb suspension (HLS) test of the 3 groups showed a significant difference $(F=898.345$, $\mathrm{p}<0.01$ ); the lower the scores, the lower the muscle powers. The control groups had significantly higher scores than the other 2 groups, and the $\mathrm{CP}+\mathrm{Sb}$ groups had significantly higher scores than the $\mathrm{CP}$ groups $(\mathrm{p}<0.01)$ (Table 8).The results showed that $\mathrm{S}$. boulardii improved muscle strength and muscle tension but did not improve the neurological deficits of hemiplegic spastic $\mathrm{CP}$ rats. 

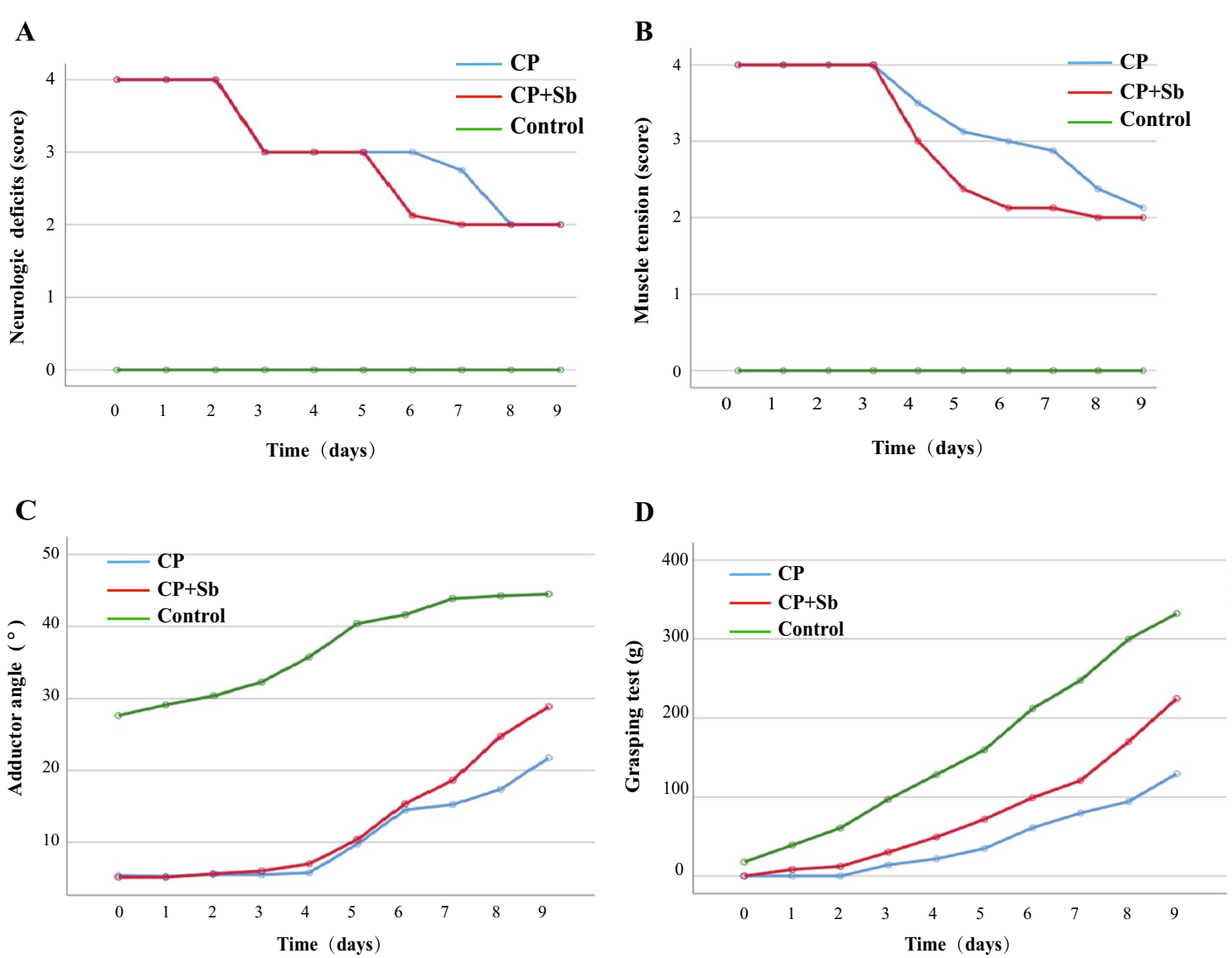

Fig. 4 A Daily changes in neurological deficits were observed. B Daily changes of grasping test. C Daily changes of muscle tension. D Daily changes of adductor angle. $n=6$ in each group

Table 5 Two-factor repeated-measures ANOVA of muscle tension

\begin{tabular}{lrrlll}
\hline & \multicolumn{1}{l}{ SS } & df & MS & F & p \\
\hline Group & 527.475 & 2 & 263.738 & 3098.455 & .000 \\
Error (group) & 1.787 & 21 & .085 & & \\
Time & 63.287 & 9 & 7.032 & 159.405 & .000 \\
Group * time & 36.275 & 18 & 2.015 & 45.684 & .000 \\
Error (time) & 8.337 & 189 & .044 & & \\
\hline
\end{tabular}

ANOVA analysis of variance, SS stdev square, $d f$ degree of freedom, MS mean square

*Interaction between time and group in the ANOVA

\section{S. boulardii affected behaviour and emotions} of hemiplegic spastic $\mathrm{CP}$ rats through the brain-gut axis pathway

The behaviour and emotions of hemiplegic spastic CP rats were improved after adding $\mathrm{S}$. boulardii. We hypothesized that $\mathrm{S}$. boulardii affected hemiplegic spastic $\mathrm{CP}$ rats by the brain-gut axis pathway, so we examined three
Table 6 Two-factor repeated-measures ANOVA of adductor angle

\begin{tabular}{lrrrrr}
\hline & \multicolumn{1}{l}{ SS } & \multicolumn{1}{l}{ df } & \multicolumn{1}{l}{ MS } & F & P \\
\hline Group & $34,396.825$ & 2 & $17,198.412$ & 2350.772 & .000 \\
Error (group) & 153.638 & 21 & 7.316 & & \\
Time & $10,702.954$ & 9 & 1189.217 & 467.293 & .000 \\
Group * time & 838.758 & 18 & 46.598 & 18.310 & .000 \\
Error (time) & 480.987 & 189 & 2.545 & & \\
\hline
\end{tabular}

ANOVA analysis of variance, SS stdev square, $d f$ degree of freedom, $M S$ mean square

*Interaction between time and group in the ANOVA

main aspects of the brain-gut axis, including inflammation, the HPA axis, and the gut microbiome.

\section{S. boulardii reduced inflammation in hemiplegic spastic CP} rats

Compared with control groups, IL-6 concentrations were significantly increased in $\mathrm{CP}$ groups $(\mathrm{P}<0.01)$. Compared 
Table 7 Two-factor repeated-measures ANOVA of grasping power

\begin{tabular}{lrrrrr}
\hline \multicolumn{1}{c}{ SS } & df & \multicolumn{1}{c}{ MS } & F & p \\
\hline Group & $565,531.308$ & 2 & $282,765.654$ & 1544.253 & 0.000 \\
Error (group) & 3845.275 & 21 & 183.108 & & \\
Time & $1,265,369.650$ & 9 & $140,596.628$ & 1128.740 & 0.000 \\
Group * time & $163,823.775$ & 18 & 9101.321 & 73.067 & 0.000 \\
Error (time) & $23,541.975$ & 189 & 124.561 & & \\
\hline
\end{tabular}

ANOVA analysis of variance, $S S$ stdev square, $d f$ degree of freedom, MS mean square

*Interaction between time and group in the ANOVA with CP groups, IL-6 concentrations were significantly decreased in $\mathrm{CP}+\mathrm{Sb}$ groups $(\mathrm{P}<0.05)$; There was no significant difference between control groups and $\mathrm{CP}+\mathrm{Sb}$ groups (Fig. 5A). Compared with the control groups, TNF- $\alpha$ concentrations were significantly increased in the $\mathrm{CP}$ groups $(\mathrm{P}<0.01)$. Compared with the $\mathrm{CP}$ groups, TNF- $\alpha$ concentrations were significantly decreased in the $\mathrm{CP}+\mathrm{Sb}$ groups $(\mathrm{P}<0.05)$; there was no significant difference between the control groups and the $\mathrm{CP}+\mathrm{Sb}$ groups (Fig. 5B).

Table 8 Results of one-way ANOVA

\begin{tabular}{|c|c|c|c|c|c|c|}
\hline & $\mathrm{CP}(\mathrm{M} \pm \mathrm{SD})$ & $\mathrm{CP}+\mathrm{Sb}(\mathrm{M} \pm \mathrm{SD})$ & Control $(M \pm S D)$ & $F$ & $p$ & LSD \\
\hline HLS test (s) & $62.75 \pm 10.29$ & $134.00 \pm 17.03$ & $300.00 \pm 0.00$ & 898.345 & 0.00 & $\begin{array}{l}\text { Con- } \\
\text { trol }>\mathrm{CP}+\mathrm{Sb}>\mathrm{CP}\end{array}$ \\
\hline SPT (\%) & $70.38 \pm 3.70$ & $82.63 \pm 3.93$ & $80.25 \pm 1.28$ & 32.939 & 0.00 & $\begin{array}{l}\text { Control }>C P \\
C P+S b>C P\end{array}$ \\
\hline TST (s) & $240.25 \pm 16.51$ & $179.63 \pm 11.93$ & $108.25 \pm 12.01$ & 187.439 & 0.00 & $\begin{array}{l}\mathrm{CP}>\mathrm{CP}+\mathrm{Sb}>\text { Con- } \\
\text { trol }\end{array}$ \\
\hline
\end{tabular}

ANOVA analysis of variance, $M$ mean, SD standard deviation, $H L S$ hind limb suspension, SPT sucrose preference test, TST tail suspension test, LSD post hoc test

\section{A}

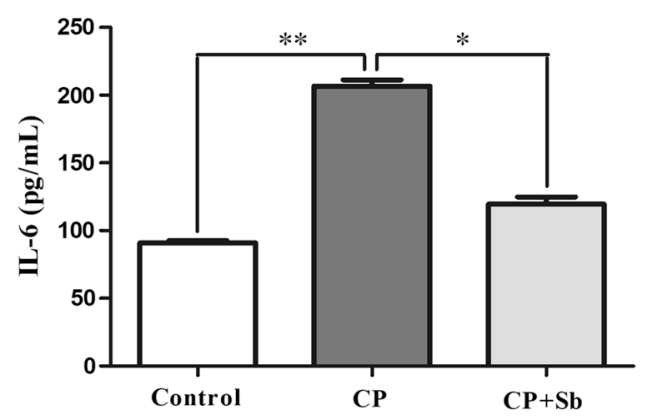

C

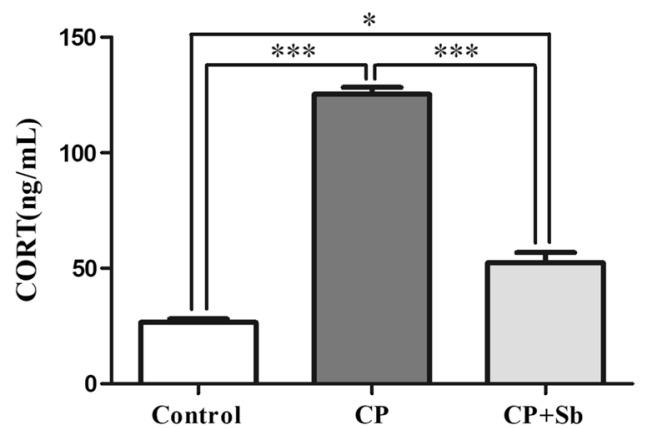

\section{B}

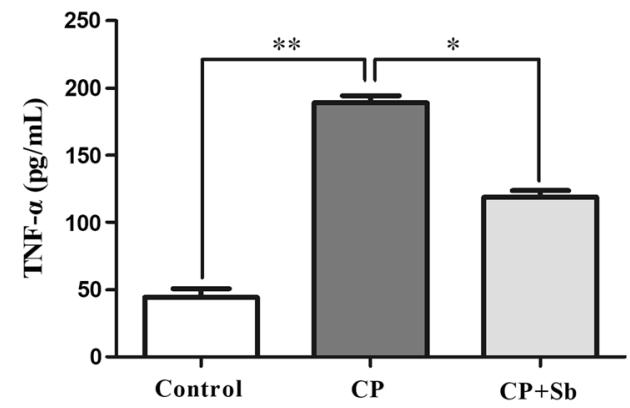

D

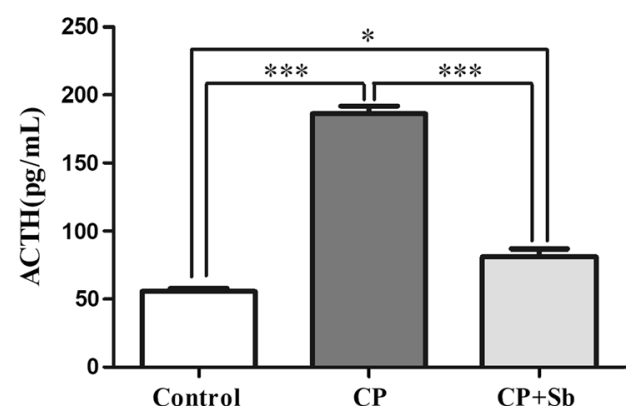

Fig. 5 Effects of S. boulardii on CP model rat plasma IL-6, TNF-a, CORT, ACTH concentrations in trunk blood. A Titre (ELISA) of IL-6 (pg/mL) in the blood on the last day after the operation between the 3 groups of rats. B Titre (ELISA) of TNF-a $(\mathrm{pg} / \mathrm{mL}$ ) in the blood on the last day after the operation between the 3 groups of rats. C Titre (ELISA) of CORT $(\mathrm{ng} / \mathrm{mL})$ in the blood on the last day after the operation between the 3 groups of rats. DTitre (ELISA) of ACTH (pg/mL) in the blood on the last day after the operation between the 3 groups of rats. Error bars represent means $\pm S E M,{ }^{*} p \leq 0.05,{ }^{* *} p \leq 0.01 .{ }^{* * *} p \leq 0.00, n=6$ in each group 


\section{S. boulardii reduced hyperactivity of the HPA axis in hemiplegic spastic CP rats}

Compared with the control groups, CORT concentrations were significantly increased in the $\mathrm{CP}$ groups $(\mathrm{P}<0.001)$ and in the $\mathrm{CP}+\mathrm{Sb}$ groups $(\mathrm{P}<0.01)$. Compared with the $\mathrm{CP}$ groups, CORT concentrations were significantly decreased in the $\mathrm{CP}+\mathrm{Sb}$ groups $(\mathrm{P}<0.001$, Fig. $5 \mathrm{C}$ ). Compared with the control groups, ACTH concentrations were significantly increased in the $\mathrm{CP}$ groups $(\mathrm{P}<0.001)$ and in the $\mathrm{CP}+\mathrm{Sb}$ groups $(\mathrm{P}<0.01)$. Compared with the $\mathrm{CP}$ groups, ACTH concentrations were significantly decreased in the $\mathrm{CP}+\mathrm{Sb}$ groups $(\mathrm{P}<0.001$, Fig. 5D).

\section{S. boulardii altered the gut microbiome of hemiplegic spastic $\mathrm{CP}$ rats}

$\alpha$-Diversity: There was no significant difference in the Chao index among the 3 groups (Fig. 6A). The Simpson index value in the control groups was significantly different from the Simpson index value in the $\mathrm{CP}+\mathrm{Sb}$ groups $(\mathrm{P}<0.01)$ and the $\mathrm{CP}$ groups $(\mathrm{P}<0.05)$. There was no significant difference in the Simpson index value between the $\mathrm{CP}$ groups and $\mathrm{CP}+\mathrm{Sb}$ groups (Fig. $6 \mathrm{~B}$ ). $\beta$-Diversity: PCA showed that there were no significant differences among the 3 groups (Fig. 6C). PLS-DA analysis showed that the 3 groups could be distinguished and clustered into three groups (Fig. 6D). The heat map of the species population showed the species abundance of each sample at the genus level. In longitudinal clustering, the closer the species are, the more similar the abundance changes of the representative species are. In horizontal clustering, the closer the samples are, the closer the trend of the representative species changes, and the abundance changes trend in the 3 groups of samples are not obvious (Fig. 6E). A Venn diagram showed that the shared OTU rates in the 3 groups were over 90\% (Fig. 6F).

The Circos diagram reflected the distribution ratio of dominant species in each sample and the distribution ratio of each dominant species in different samples. At the genus level, Lactobacillus was dominant in the $\mathrm{CP}+\mathrm{Sb}$ group. Prevotella was dominant in the control groups. Bacteroides was predominantly distributed in the $\mathrm{CP}$ groups (Fig. 7A). The rank sum test was used to perform hypothesis tests on species that show differences in abundance in different groups of microbial communities to assess the significance of the observed differences. At the phylum level, there were differences in Campylobacterota, Proteobacteria, Desulfobacterota, Elusimicrobiota, and Chloroflexi. At the family level, there were significant differences in Prevotellaceae, Lachnospiraceae, Oscillospiraceae, Staphylococcaceae, Helicobacteraceae, Clostridiaceae, Desulfovibrionaceae, Elusimicrobiaceae, Butyricicoccaceae, Anaerovoracaceae, Bacteroidaceae, Lactobacillaceae, Muribaculaceae, Ruminococcaceae, Acidaminococcaceae, Peptostreptococcaceae, and Erysipelotrichaceae. At the genus level, there were differences in Prevotella, Ruminococcus, Blautia, Staphylococcus, Helicobacter, and Dorea (Fig. 7B).

\section{Discussion}

In 2006, "The Definition and Classification of Cerebral Palsy, April 2006," further defined CP [37]. By considering the definition of $\mathrm{CP}$, we can draw the following conclusions: (1) CP occurs in the immature and developing brain, (2) it can occur in prenatal, perinatal and postpartum. By means of a comparative study of experimental data from the literature, the P12-13 rat pup cerebral cortex corresponds to that of the full-term new born human infant with regard to the degree of maturation[38]. The rat cortex reaches approximately $90 \%$ of its adult weight by P20. In humans, brain weight reaches a similar plateau by $2-3$ years of age. Thus, based on brain weights alone, P20 in rats appears to correspond to a 2-3 year old human child $[39,40]$. The brain of P17 rats is in the development stage, which equivalent to a human infant or analogous to the human toddler [41-43]. So insults performed at P17 occur in the ideal window to induce CP.

Compared with the most rodent models used smaller day old rats do not show an obvious CP-like motor phenotype, the 17 day old rat model showed typical CPlike motor phenotype, which was considered to maybe related to the following reasons [35, 36, 44, 45]. Despite the fact that impairments of the Corticospinal tract (CST) are widely recognized as the underlying cause of CP, most studies simply focus on brain development (or maturation of oligodendrocytes) rather than trying to time injuries in animal models to parallel the timing of corticospinal tract development in human neonates. The CST controlled human motor function, which is

(See figure on next page.)

Fig. 6 Changes of intestinal microbiota in three groups. A The Chao index analysis showed that there was no difference between the 3 groups. B The Simpson index analysis between 3 groups. C PCA showed that there was no significant difference between the 3 groups. D PLS-DA analysis showed that the 3 groups could be distinguished and clustered into three groups. $\mathbf{E}$. The heatmap of the species population shows the species abundance of each sample at the genus level. $\mathbf{F}$ Venn diagram analysis showed the shared and unique OTUs in the 3 groups. Error bars represent means $\pm S E M,{ }^{*} p \leq 0.05,{ }^{* *} p \leq 0.01, n=6$ in each group. 'Con' instead of 'Control,',Sb'instead of 'CP $+S b^{\prime}$ 


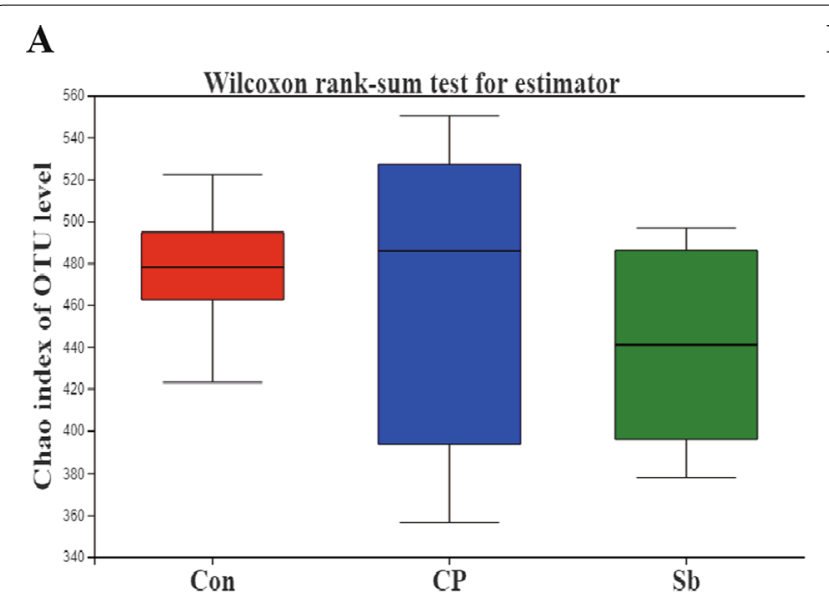

B

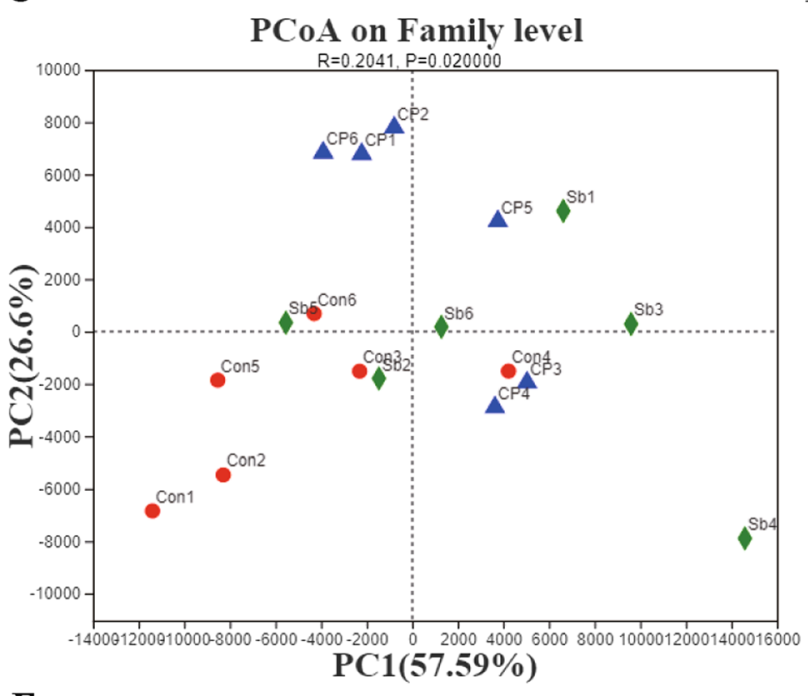

D
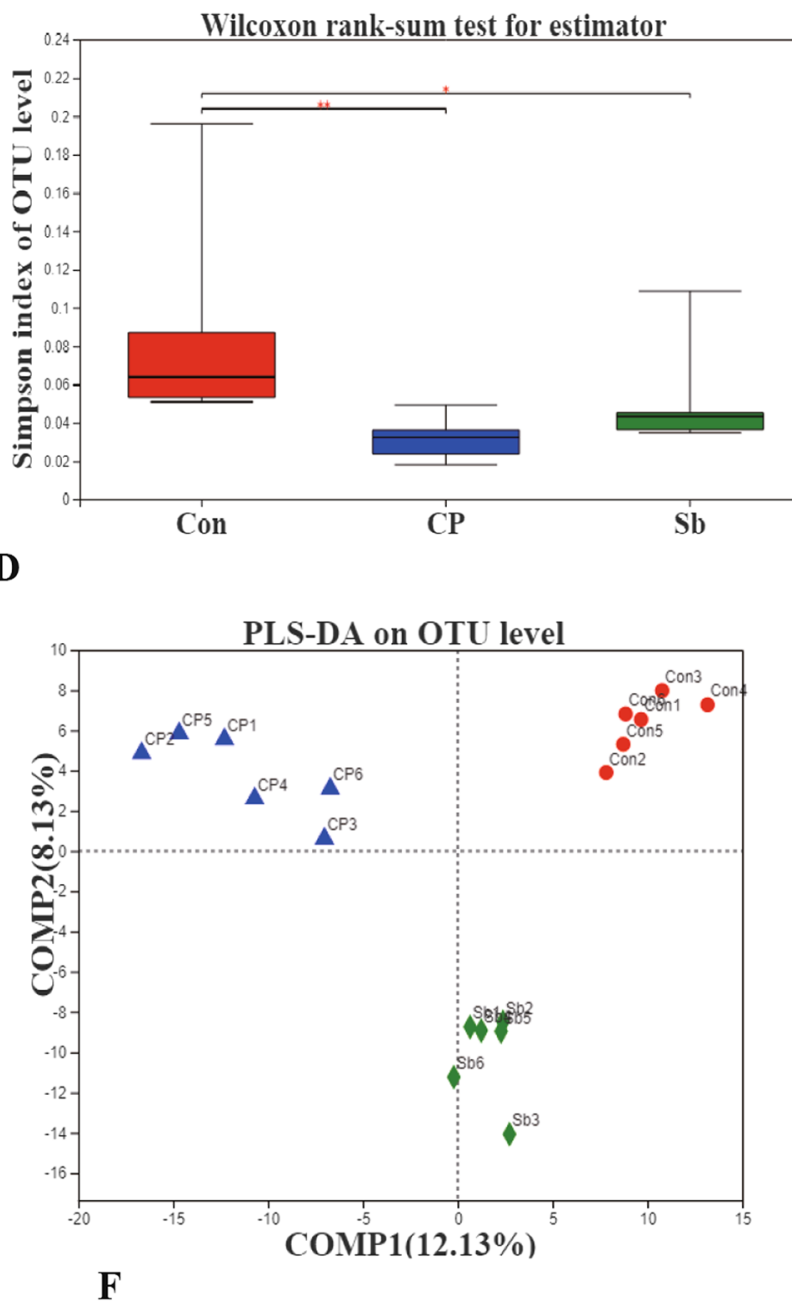

$\mathbf{E}$

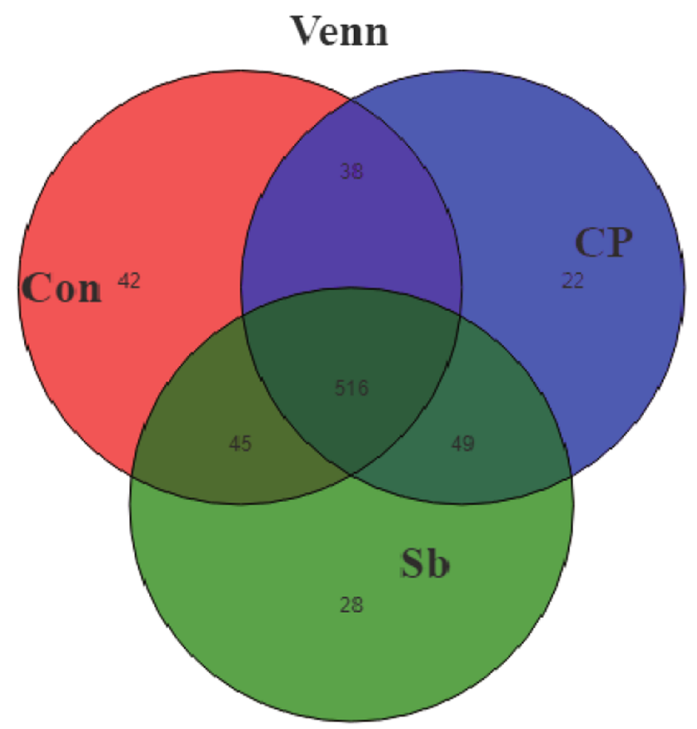

Fig. 6 (See legend on previous page.) 


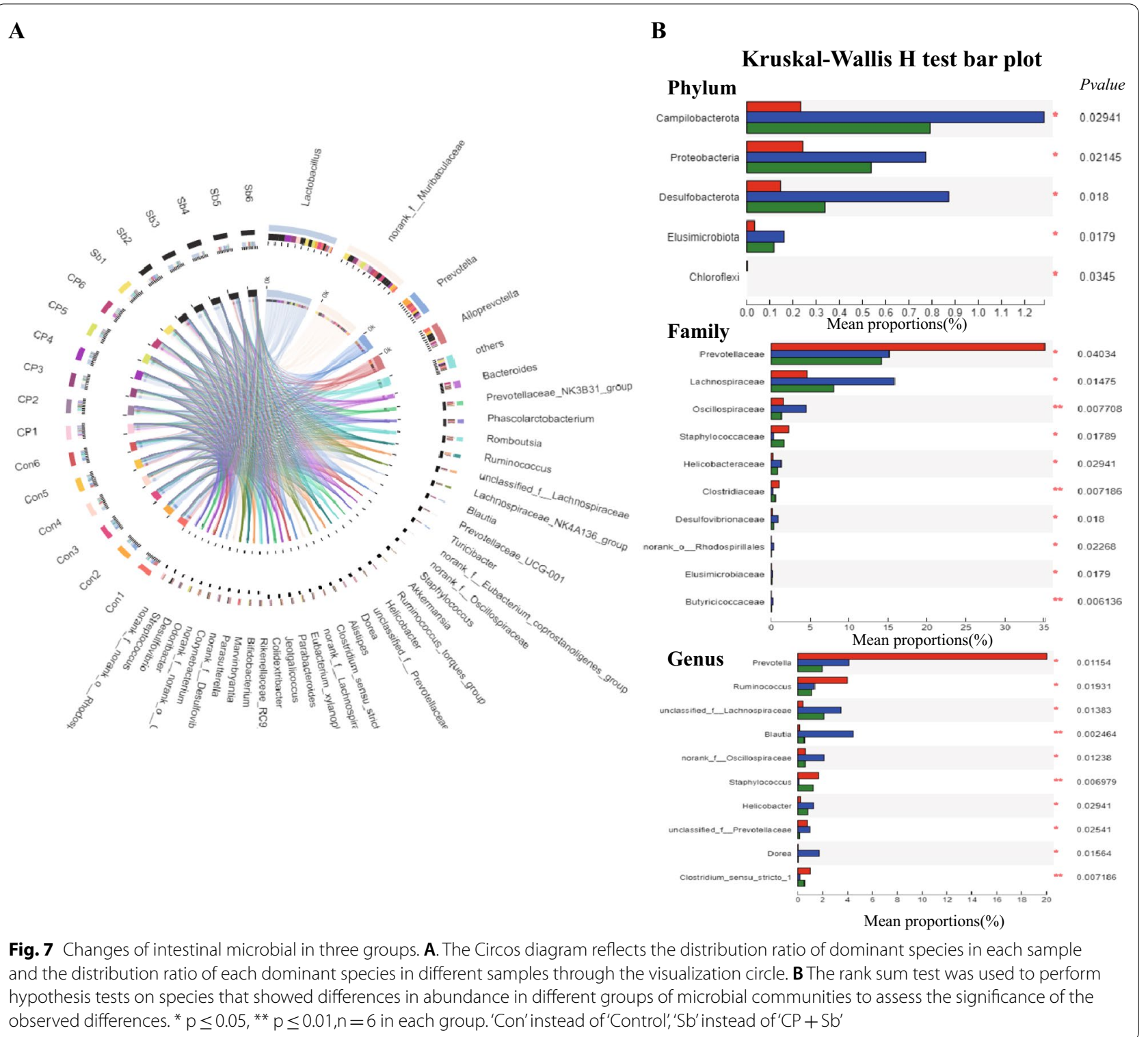

necessary for recovery of motor function in patients with brain injury [46]. Martin rose 3 developmental periods of the human brain CST. First, develop of axons of cortical lamina 5 neurons during the perinatal. Next, refined the grey matter terminations of the spinal cord within 2 years after born(postnatal development)[47]. It is well known that the younger the age, the greater the neural plasticity. Although the brain of 17 day old rats is still in the development stage, the neural plasticity is significantly lower than smaller day old rats.

Based on the clinical history, MRI scan findings and standardized neuromotor assessment were used to diagnose CP [1]. The most prominent anatomical feature of spastic CP was the lesion of the motor cortex and the subcortical white matter [2, 48]. White matter tracts from both the somatosensory and motor cortex play an important role in the pathophysiology of motor disability in CP patients [49]. The CST originates from several cortical areas, and approximately half of these axons extend from neurons in the primary motor cortex [50]. Damaging the CST can reduce inhibitory tone and contribute to hypertonia. In $90 \%$ of cases of CP, MRI scans of the brain showed abnormal findings [48]. Regarding the standardized neuromotor assessment, Novak reported that combinations of standardized individually administered neurological and motor assessment tools 
were recommended [51]. Therefore, we performed unilateral lesioning of the brain motor cortex and designed this model to simulate hemiplegic spastic $\mathrm{CP}$ as closely as possible. At the same time, the rats underwent MRI scans, and we found damage to the motor cortex and subcortical white matter, which was consistent with the imaging characteristics of spastic CP. Neuromotor assessments included muscle strength, muscle tension, and neurological deficits, and our results showed that the spastic CP rat model followed the standard of human spastic CP.

Shevell explored the argument for a consideration to view $C P$ as a spectrum disorder because children with $\mathrm{CP}$ also had many associated conditions such as depression $[52,53]$. Depressive symptoms in children and adults with spastic CP were much more common [54-56]. A multidisciplinary approach offered the best model for the medical care of children with CP to manage various conditions as well as psychosocial needs $[57,58]$. To the best of our knowledge, this is the first rodent model to focus on depression-like behaviour of CP. Compared with the control groups, the CP groups showed depression-like behaviour in the tail suspension test and sucrose preference test in our study. Inflammation has been increasingly recognized as an important contributor to CNS injury $[59,60]$. CP and depression-like behaviour have been linked to inflammation [61]. Epidemiologic studies have linked elevated cytokines in the umbilical cord, amniotic fluid, and foetal blood to CP [32]. Increased pro-inflammatory cytokines in neonatal blood during the first few days in the infant after birth had 100\% sensitivity and specificity in the prediction of $\mathrm{CP}$ in late preterm and term infants [62]. Depression is associated with both a chronic low-grade inflammatory response, activation of cell-mediated immunity, and many inflammatory disorders, and neuroinflammatory disorders might trigger clinical depression-like behaviour [59]. The new and emerging discoveries that linked inflammation with neuropsychopathology provided opportunities for novel therapeutics. In this experiment, the inflammatory factors of $\mathrm{CP}$ rats were higher than the inflammatory factors of the control group, and the relationship between inflammatory factors and depression-like behaviour can explain the depression-like behaviour of CP rats.

The gut-brain axis is emerging as a particular area of interest and a potential new therapeutic target for the effective treatment of CNS disorders [63, 64]. The relationship between the gut-brain axis and the CNS was mainly through the gut microbiota, immune system, and neuroendocrine pathway. Long-term chronic HPA imbalance harms the brain [62]. A hyperactive HPA axis is often associated with clinical depression-like behaviour [65]. The beneficial effects of probiotic consumption on behaviour and brain function are now becoming increasingly appreciated in a variety of inflammatory diseases $[66,67]$. A probiotic-induced reduction in CORT and ACTH levels was observed in chronic stress rats $[68,69]$. Our study showed that the increase of gut microbiota diversity and species richness in the $\mathrm{CP}+$ Sb groups compared with CP groups; the levels of inflammatory, and $\mathrm{ACTH}$ and CORT in the $\mathrm{CP}+\mathrm{Sb}$ groups were significantly lower than in $\mathrm{CP}$ groups $(\mathrm{p}<0.01)$, indicating that $\mathrm{S}$. boulardii can reduce the inflammation in $\mathrm{CP}$ rats and downregulate a hyperactive HPA axis, and improve the composition of gut microbiota, the results were which were consistent with our previous hypothesis.

\section{Limitations}

There were several limitations of this study. First, there was insufficient pathological evidence gathered. Second, the behavioural tests we chose did not fully reflect the cognition, memory, and sensation of rats, and extensive testing on behaviour was necessary for detailed evaluation. We were aware that many argued that rat models were insufficient because of differences from humans in that similar injuries did not lead to similar phenotypes, and it seemed that matching based on phenotype alone was insufficient as a model, as the incidence of $\mathrm{CP}$ caused by brain injury was low, whereas brain trauma and hypoxia-ischaemia in rats shared many homologous pathological mechanisms [70]. This study might also be suitable for the study of CP caused by hypoxia-ischaemia, but this aspect also required further investigation. There have been no reports about the application of probiotics for emotional regulation in children or adults with CP. We found relevant literature such as clinical trials to analyse the efficacy of a probiotic in the treatment of constipation in children with CP [71]. A follow-up study of probiotic effects in very preterm infants found that probiotics can reduce necrotizing enterocolitis [72]. In the future, we hope that probiotics could be applied to children with $\mathrm{CP}$ in the role of emotion regulation based on a large number of experiments. There are big gaps in information about the interraction between the microbiome and their parasitifer, and the pathway of its metabolites and so on. Recently, the combinations of gas chromatography and liquid chromatography have been used in metabolomics studies to achieve sensitive and accurate metabolic profiling [73]. The use of gas chromatography and liquid chromatography is a possible area of future work to better identify the metabolic changes and pathways involved. 


\section{Conclusions}

The model developed in this study mimics a hemiplegic spastic cerebral palsy. We destroyed the right-brain motor cortex and the cingulate cortex by resecting the cortex and subcortical white matter of 17-day-old SD rats, which led to the hemiplegic spastic CP-like phenotype. The hemiplegic spastic $\mathrm{CP}$ rats exhibited symptoms of typical paralysis, high muscle tension, and lower muscle strength. Three months later, the right hind paws of hemiplegic spastic CP rats were flexed, and because of lower muscle strength, the gaits of the right lower limbs were abnormal during walking. The hemiplegic spastic $\mathrm{CP}$ rats had depression-like behaviour. S. boulardii could improve the behaviour and emotional conditions of hemiplegic spastic CP rats through the gut-brain axis pathway to reduce inflammation, downregulate the HPA axis, and improve the gut microbiota. Our results indicated that $\mathrm{S}$. boulardii might have potential in treating hemiplegic spastic $\mathrm{CP}$ rat models or as an add-on therapy. More interestingly, these effects might be accompanied by positive modulatory actions on depression and certain protective effects in a hemiplegic spastic $\mathrm{CP}$ rat model.

\section{Methods}

\section{Ethical statement}

All of the experimental procedures were in accordance with the "Guidance Suggestions for the Care and Use of Laboratory Animals" issued by the Ministry of Science and Technology of China. All the studies were approved by the Ethics Committee of Jiamusi University. This study was carried out in compliance with the ARRIVE guidelines.

\section{Animals}

All animals for the experiment were obtained from the Specific Pathogen Free Animal Laboratory in Harbin Medical University. A total of 30 specific pathogen-free grade SD rats (male and female), aged 17 days and weighing 28-30 g, were randomly assigned to control groups $(n=6)$ or CP groups $(n=24)$. The rats were housed in a feeding room at $22 \pm 1{ }^{\circ} \mathrm{C}$ and $50-70 \%$ humidity with a $12 \mathrm{~h}$ light/dark cycle[74].

\section{Modelling of spastic CP}

Food and water were withheld from the rats for $3 \mathrm{~h}$ before the operation. After anaesthetization (1\% pentobarbital sodium at $40 \mathrm{mg} / \mathrm{kg}$ ), the rats were fixed on a stereotactic apparatus (Anhui Zhenghua Biologic Apparatus Facilities Co., Ltd., Huaibei, Anhui Province, China) [75]. Referring to the Atlas of the Neonatal Rat Brain, the parietal incision about 2-cm-long was cut on the right side of the median line through the skin, subcutaneous tissue, deep fascia and periosteum, layer by layer. After removal of the periosteum, the bregma and sagittal suture were exposed. Then, a bone drill was used to drill holes with a range of $0.5 \times 0.5 \mathrm{~cm}$ which was on the right side of the median line and in front of the bregma. The motor cortex was exposed by cutting and lifting a flap of bone and then a size 15 scalpel bladeaspirated was inserted into the brain cortex to cut the cortex, and the cut off brain tissue were brought out through a pipette. The area of the cerebral cortex we cut was $0.5 \times 0.5$, and the depth was $0.5-0.6 \mathrm{~cm}$. Cotton balls with saline were used for hemostasis by compression. After haemostasis, the wound was rinsed with normal saline and sewed after filling with a gelatine sponge $[75,76]$.

\section{Magnetic resonance imaging (MRI) of the spastic CP model} The rats were anaesthetized with $3.5 \%$ isoflurane mixed with oxygen. The rats were placed in the animal bed in the supine position and kept warm with a water-bath mat. During scanning, the rats were anaesthetized with $0.8 \% \sim 1 \%$ isoflurane. Vital signs were observed by respiratory monitoring, and the respiratory rate of the rats was maintained at $30 \pm 5$ times $/ \mathrm{min}$. The image was scanned by an Agilent Technologies 9.4 t/400 ps animal scanner (Agilent Technologies, Santa Clara, CA) with an aperture of $40 \mathrm{~cm}$ and a gradient coil of $26 \mathrm{~cm}$. The structural image was obtained by rapid acquisition with the relaxation enhancement sequence. The relevant parameters are as follows: repetition time $=8000 \mathrm{~ms}$, echo time $=18 \mathrm{~ms}$, field of vision $=16 \mathrm{~mm} \times 16 \mathrm{~mm}$, matrix $=192 \times 192$, layer thickness $=0.5 \mathrm{~mm}$, bandwidth $=50 \mathrm{kHz}$.

\section{S. boulardii preparation and measurement of physiological variables}

After finishing the MRI, the rats in the model group were randomly divided into two groups: with $(\mathrm{CP}+\mathrm{Sb}$ group) and without $\mathrm{S}$. boulardii (CP group). Rats in the $\mathrm{CP}+\mathrm{Sb}$ group were administered $\mathrm{S}$. boulardii $\left(10^{7} \mathrm{CFU} /\right.$ day, total $0.2 \mathrm{~mL}$ ), while rats in the $\mathrm{CP}$ group were administered equal amounts of normal saline by gavage for 9 days. We observed the weight, water content of faeces, and general states of rats every day. Each rat was placed in a clean cage alone at 8-9 am every day to collect faeces. Tubes were weighed to obtain the total weight of the stools, and then the stools were dried overnight and reweighed. The stool water content was calculated from the difference between the total and dry weights. Referring to some items of the National Institutes of Health Stroke Scale and diarrhoea index, we improved the scoring strategies and obtained the general state scores (Additional file 1). Scores in each category were summarized to generate an overall general state score. 
Behavioural evaluation of the model of spastic CP

Hypertonic and motor disturbances of spastic CP were assessed by neurological deficits, grasping test, HLS test, and muscle tension and adductor angle. Depression in rats was evaluated using the TST and SPT. Behaviour tests were conducted every day and analysed in a blind fashion (except the HLS test, TST and SPT, which were given only on the last day). For detailed behavioural examination methods, see Additional file 2.

\section{S rRNA of the model of spastic CP Sample collection}

After the test on the last day, a $1.5 \mathrm{~mL}$ EP tube was used to collect 1-2 pieces of fresh faeces from each rat, which was sent for examination immediately after being frozen in liquid nitrogen, and the rats were anesthetized with pentobarbital (70 $\mathrm{mg} / \mathrm{kg}$, i.p.) and then euthanized by decapitation.

\section{DNA extraction and PCR amplification}

Microbial community genomic DNA was extracted from faecal samples using the E.Z.N.A. ${ }^{\circledR}$ soil DNA Kit (Omega Bio-tek, Norcross, GA, U.S.) according to the manufacturer's instructions. The DNA extract was checked on a $1 \%$ agarose gel, and DNA concentration and purity were determined with a NanoDrop 2000 UV-vis spectrophotometer (Thermo Scientific, Wilmington, USA)[77]. The hypervariable region $\underline{\mathrm{V} 3-\mathrm{V} 4}$ of the bacterial $16 \mathrm{~S}$ rRNA gene was amplified with primer pair $338 \mathrm{~F}$ (5'-ACTCCT ACGGGAGGCAGCAG-3') and 806R (5'-GGACTA CHVGGGTWTCTAAT-3') by an ABI GeneAmp ${ }^{\circledR} 9700$ PCR thermocycler (ABI, CA, USA). PCR amplification of the 16S rRNA gene was performed as follows: initial denaturation at $95{ }^{\circ} \mathrm{C}$ for $3 \mathrm{~min}$, followed by 27 cycles of denaturation at $95{ }^{\circ} \mathrm{C}$ for $30 \mathrm{~s}$, annealing at $55^{\circ} \mathrm{C}$ for $30 \mathrm{~s}$ and extension at $72{ }^{\circ} \mathrm{C}$ for $45 \mathrm{~s}$, and a single extension at $72{ }^{\circ} \mathrm{C}$ for $10 \mathrm{~min}$, ending at $10{ }^{\circ} \mathrm{C}$. The PCR mixtures contained $5 \times$ TransStart FastPfu buffer, $4 \mu \mathrm{L}, 2.5 \mathrm{mM}$ dNTPs, $2 \mu \mathrm{L}$, forward primer $(5 \mu \mathrm{M}), 0.8 \mu \mathrm{L}$, reverse primer $(5 \mu \mathrm{M}), 0.8 \mu \mathrm{L}$, TransStart FastPfu DNA Polymerase, $0.4 \mu \mathrm{L}$, template DNA, $10 \mathrm{ng}$, and $\mathrm{ddH}_{2} \mathrm{O}$ up to 20 $\mu \mathrm{L}$. PCRs were performed in triplicate. The PCR product was extracted from a $2 \%$ agarose gel and purified using the AxyPrep DNA Gel Extraction Kit (Axygen Biosciences, Union City, CA, USA) according to the manufacturer's instructions and quantified using a Quantus ${ }^{\mathrm{TM}}$ Fluorometer (Promega, USA) [78, 79].

\section{Illumina MiSeq sequencing}

Purified amplicons were pooled in equimolar concentrations and paired-end sequenced $(2 \times 300)$ on an Illumina MiSeq platform (Illumina, San Diego, USA) according to the standard protocols by Majorbio Bio-Pharm
Technology Co. Ltd. (Shanghai, China). The raw reads were deposited into the NCBI Sequence Read Archive (SRA) database (http://www.ncbi.nlm.nih.gov/Traces/ sra). The data files were deposited at NCBI SRA database under project accession no. PRJNA729263.

\section{Processing of sequencing data}

The raw $16 \mathrm{~S}$ rRNA gene sequencing reads were demultiplexed, quality filtered by Trimmomatic and merged by FLASH with the following criteria: (i) the 300 bp reads were truncated at any site receiving an average quality score of $<20$ over a 50 -bp sliding window, and the truncated reads shorter than $50 \mathrm{bp}$ and reads containing ambiguous characters were discarded. (ii) Only overlapping sequences longer than $10 \mathrm{bp}$ were assembled according to their overlapping sequence, with a maximum mismatch ratio of 0.2 in the overlap region; reads that could not be assembled were discarded, and (iii) Samples were distinguished according to the barcode and primers, and the sequence direction was adjusted with exact barcode matching and 2-nucleotide mismatch in primer matching.

Operational taxonomic units (OTUs) with 97\% similarity cutoff were clustered using UPARSE (version 7.1, http://drive5.com/uparse/), and chimaeric sequences were identified and removed. The data were analysed on the free online platform of the Majorbio Cloud Platform (www.majorbio.com).

\section{Serum inflammatory cytokine and HPA axis hormone measurements}

Blood samples were collected in a plastic tube. The serum was obtained by centrifugation $(3000 \mathrm{~g}, 10 \mathrm{~min}$, and $4{ }^{\circ} \mathrm{C}$ ) and stored at $-80{ }^{\circ} \mathrm{C}$. To avoid fluctuations in the results owing to the circadian rhythm of the HPA axis, samples in each group were collected at the same time of day (between 8:00 and 10:00 h). Serum concentrations of IL-6, TNF- $\alpha$, ACTH and CORT were measured in duplicate using a commercial ELISA kit according to the manufacturer's specifications. The plates were read at $450 \mathrm{~nm}$ using an ELISA reader. The sensitivity of the IL- 6 kit was $43 \mathrm{pg} / \mathrm{ml}$. The sensitivity of the TNF- $\alpha$ kit was $9.1 \mathrm{pg} / \mathrm{ml}$, the sensitivity of the ACTH kit was $6 \mathrm{pg} / \mathrm{ml}$, and the sensitivity of the CORT kit was $0.3 \mathrm{ng} / \mathrm{ml}$.

\section{Statistical analysis}

Data were analysed using IBM SPSS for Windows 24.0 software (SPSS Inc., Chicago, IL, USA). All results were given as the mean \pm SEM. Data from the tail suspension test, hind limb suspension test and sucrose preference test were analysed using one-way ANOVA (normally distributed) followed by Bonferroni post hoc tests. Data 
including body weight, faecal water content, general state, muscle strength, muscle tension, adductor angle, and neurological deficits were analysed by two-factor repeated-measures ANOVA. P values less than or equal to 0.05 were considered statistically significant. Before the comparison, the Shapiro-Wilk test was used to verify the compliance of numerical variables with the normal distribution. All variables were found to be normally distributed.

\author{
Abbreviations \\ CP: Cerebral palsy; ELISA: Enzyme-linked immunosorbent assay; TNF-a: \\ Tumour necrosis factor-a; IL-6: Interleukin-6; HPA: Hypothalamic-pituitary- \\ adrenal; ACTH: Adrenocorticotropic hormone; CORT: Corticosterone; CNS: \\ Central nervous system; MIA: Maternal immune activation; MRI: Magnetic reso- \\ nance imaging; TST: Tail suspension test; SPT: Sucrose preference test; OTU: \\ Operational taxonomic units; CTS: Corticospinal tract.
}

\section{Supplementary Information}

The online version contains supplementary material available at https://doi. org/10.1186/s12868-021-00679-4.

\section{Additional file 1. General state scores.}

Additional file 2. Behavioural examination methods.

Additional file 3: Table S1. Two-factor repeated measures ANOVA of body weight. Table S2. Multiple comparisons of body weight. Table S3. Two-factor repeated measures ANOVA of fecal water content. Table S4. Multiple comparisons of fecal water content. Table S5. Two-factor repeated measures ANOVA of general state scores. Table S6. Multiple comparisons of gerenal state scores. Table S7. Two-factor repeated measures ANOVA of neurological deficits. Table S8. Multiple comparisons of neurologic deficits. Table S9. Two-factor repeated measures ANOVA of muscle tension. Table S10. Multiple comparisons of muscle tension. Table S11. Two-factor repeated measures ANOVA of adductor angle. Table S12. Multiple comparisons of adductor angle. Table S13. Twofactor repeated measures ANOVA of grasping power. Table S14. Multiple comparisons of grasping test.

\section{Acknowledgements}

Not applicable.

\section{Authors' contributions}

DT: Methodology, Software, Data curation, Writing-Original draft preparation. TZ: Visualization, Writing-Reviewing and Editing, Investigation. XL: Conceptualization, Supervision. WP: Software, Validation. All authors read and approved the final manuscript.

\section{Funding}

This research did not receive any specific grant from funding agencies in the public, commercial, or not-for-profit sectors.

\section{Availability of data and materials}

The datasets generated and analysed during the current study are available in the [https://submit.ncbi.nlm.nih.gov/subs/bioproject/.] repository. The data files were deposited at NCBI SRA database under project accession no. PRJNA729263.

\section{Declarations}

Ethics approval and consent to participate

All of the experimental procedures were in accordance with the "Guidance Suggestions for the Care and Use of Laboratory Animals" issued by the
Ministry of Science and Technology of China. All the studies were approved by the Ethics Committee of Jiamusi University.

\section{Consent for publication}

Not applicable.

\section{Competing interests}

None.

\section{Author details}

${ }^{1}$ College of Basic Medicine, Jiamusi University, Jiamusi, Heilongjiang, China

${ }^{2}$ Jiamusi Central Hospital, Jiamusi, Heilongjiang, China. ${ }^{3}$ College of Rehab Medicine, Jiamusi University, Jiamusi, China. ${ }^{4}$ Rehab Center for Child Cerebral Palsy, Jiamusi, Heilongjiang, China. ${ }^{5}$ Institute of Pediatric Neurological Disorders, Jiamusi University, Jiamusi, China.

Received: 21 January 2021 Accepted: 23 November 2021

Published online: 07 December 2021

\section{References}

1. Graham HK, Rosenbaum P, Paneth N, Dan B, Lin JP, Damiano DL, Becher JG, Gaebler-Spira D, Colver A, Reddihough DS, Crompton KE, Lieber RL. Cerebral palsy. Nat Rev Dis Primers. 2016;2:15082. https://doi.org/10. 1038/nrdp.2015.82.

2. Colver A, Fairhurst C, Pharoah PO. Cerebral palsy. Lancet. 2014;383(9924):1240-9.

3. Reddihough DS, Collins KJ. The epidemiology and causes of cerebral palsy. Aust J Physiother. 2003;49(1):7-12.

4. Durkin MS, et al. Prevalence of cerebral palsy among 8-year-old children in 2010 and preliminary evidence of trends in its relationship to low birthweight. Paediatr Perinat Epidemiol. 2016;30(5):496-510.

5. Surman G, et al. UKCP: a collaborative network of cerebral palsy registers in the United Kingdom. J Public Health (Oxf). 2006;28(2):148-56.

6. Shikako-Thomas K, et al. Quality of life from the perspective of adolescents with cerebral palsy: "I just think I'm a normal kid, I just happen to have a disability." Qual Life Res. 2009;18(7):825-32.

7. Woodgate RL, et al. Intense parenting: a qualitative study detailing the experiences of parenting children with complex care needs. BMC Pediatr. 2015;15:197.

8. Michelsen SI, et al. Education and employment prospects in cerebral palsy. Dev Med Child Neurol. 2005;47(8):511-7.

9. Maestro-Gonzalez A, et al. Quality of life as assessed by adults with cerebral palsy. PLoS ONE. 2018;13(2):e0191960.

10. Nguyen TL, Nguyen HP, Nguyen TK. The effects of bone marrow mononuclear cell transplantation on the quality of life of children with cerebral palsy. Health Qual Life Outcomes. 2018;16(1):164.

11. Colver A, Fairhurst C, Pharoah PO. Cerebral palsy. Lancet. 2014;383(9924):1240-9. https://doi.org/10.1016/S0140-6736(13)61835-8.

12. Shankaran S. Prevention, diagnosis, and treatment of cerebral palsy in near-term and term infants. Clin Obstet Gynecol. 2008;51(4):829-39.

13. Nolan KW, Cole LL, Liptak GS. Use of botulinum toxin type $A$ in children with cerebral palsy. Phys Ther. 2006;86(4):573-84.

14. Dekopov AV, et al. Neurosurgery of the spasticity syndrome in children cerebral palsy. Zh Nevrol Psikhiatr Im S S Korsakova. 2012;112(7 Pt 2):34-40.

15. Ait-Belgnaoui A, et al. Prevention of gut leakiness by a probiotic treatment leads to attenuated HPA response to an acute psychological stress in rats. Psychoneuroendocrinology. 2012;37(11):1885-95.

16. Čitar M, et al. Human intestinal mucosa-associated Lactobacillus and Bifidobacterium strains with probiotic properties modulate IL-10, IL-6 and IL-12 gene expression in THP-1 cells. Benef Microbes. 2015;6(3):325-36.

17. Peirce JM, Alviña K. The role of inflammation and the gut microbiome in depression and anxiety. J Neurosci Res. 2019;97(10):1223-41.

18. de Avila LF, et al. Modulation of IL-12 and IFNy by probiotic supplementation promotes protection against Toxocara canis infection in mice. Parasite Immunol. 2016;38(5):326-30.

19. de Avila LF, et al. Saccharomyces boulardii reduces infection intensity of mice with toxocariasis. Vet Parasitol. 2012;187(1-2):337-40. 
20. Cardoso PD, et al. Saccharomyces boulardii reduces the mean intensity of infection in mice caused by the consumption of liver contaminated by Toxocara canis. Parasitol Res. 2020;119(3):1161-5.

21. Vermeersch, S.J., et al., Economic evaluation of S. boulardii CNCM I-745 for prevention of antibioticassociated diarrhoea in hospitalized patients. Acta Gastroenterol Belg, 2018. 81(2): p. 269-276.

22. Ransohoff RM, et al. Neuroinflammation: ways in which the immune system affects the brain. Neurotherapeutics. 2015;12(4):896-909.

23. Deverman BE, Patterson PH. Cytokines and CNS development. Neuron. 2009;64(1):61-78.

24. Kim S, et al. Maternal gut bacteria promote neurodevelopmental abnormalities in mouse offspring. Nature. 2017;549(7673):528-32.

25. Knuesel I, et al. Maternal immune activation and abnormal brain development across CNS disorders. Nat Rev Neurol. 2014;10(11):643-60.

26. Kuban KC, et al. The breadth and type of systemic inflammation and the risk of adverse neurological outcomes in extremely low gestation newborns. Pediatr Neurol. 2015;52(1):42-8

27. Marques $\mathrm{AH}$, et al. Maternal stress, nutrition and physical activity: Impact on immune function, CNS development and psychopathology. Brain Res. 2015;1617:28-46.

28. Cryan JF, Dinan TG. Mind-altering microorganisms: the impact of the gut microbiota on brain and behaviour. Nat Rev Neurosci. 2012;13(10):701-12

29. Gracie DJ, Hamlin PJ, Ford AC. The influence of the brain-gut axis in inflammatory bowel disease and possible implications for treatment. Lancet Gastroenterol Hepatol. 2019;4(8):632-42.

30. Rice MW, Pandya JD, Shear DA. Gut microbiota as a therapeutic target to ameliorate the biochemical, neuroanatomical, and behavioral effects of traumatic brain injuries. Front Neurol. 2019;10:875.

31. Brandenburg JE, Fogarty MJ, Sieck GC. A critical evaluation of current concepts in cerebral palsy. Physiology (Bethesda). 2019;34(3):216-29.

32. Jiang $Y Q$, Zaaimi $B$, Martin JH. Competition with primary sensory afferents drives remodeling of corticospinal axons in mature spinal motor circuits. J Neurosci. 2016;36(1):193-203.

33. Smith CC, Paton JFR, Chakrabarty S. Descending systems direct development of key spinal motor circuits. J Neurosci. 2017;37(26):6372-87.

34. Song H, Cho J, Lee S. Transcortical photothrombotic pyramidotomy model with persistent motor deficits. PLoS ONE. 2018;13(12):e0204842.

35. Rumajogee $\mathrm{P}$, et al. Rodent hypoxia-ischemia models for cerebral palsy research: a systematic review. Front Neurol. 2016;7:57.

36. Fan LW, et al. Hypoxia-ischemia induced neurological dysfunction and brain injury in the neonatal rat. Behav Brain Res. 2005;165(1):80-90.

37. Krigger KW. Cerebral palsy: an overview. Am Fam Physician. 2006;73(1):91-100.

38. Romijn HJ, Hofman MA, Gramsbergen A. At what age is the developing cerebral cortex of the rat comparable to that of the full-term newborn human baby? Early Hum Dev. 1991;26(1):61-7.

39. Dekaban AS. Changes in brain weights during the span of human life: relation of brain weights to body heights and body weights. Ann Neurol. 1978;4(4):345-56.

40. Dobbing J, Sands J. Comparative aspects of the brain growth spurt. Early Hum Dev. 1979;3(1):79-83.

41. Huh JW, Raghupathi R. Chronic cognitive deficits and long-term histopathological alterations following contusive brain injury in the immature rat. J Neurotrauma. 2007;24(9):1460-74.

42. Robertson $\mathrm{CL}$, Saraswati M. Progesterone protects mitochondrial function in a rat model of pediatric traumatic brain injury. J Bioenerg Biomembr. 2015:47(1-2):43-51.

43. Anthonymuthu TS, et al. Detection of brain specific cardiolipins in plasma after experimental pediatric head injury. Exp Neurol. 2019;316:63-73.

44. Barboza R, Lima FA, Reis AS, Murillo OJ, Peixoto EPM, Bandeira CL, Fotoran WL, Sardinha LR, Wunderlich G, Bevilacqua E, Lima MRD, Alvarez JM, Costa FTM, Gonçalves LA, Epiphanio S, Marinho CRF. TLR4-mediated placental pathology and pregnancy outcome in experimental malaria. Sci Rep. 2017;7(1):8623. 2018. https://doi.org/10.1038/s41598-017-08299-x. Erratum. In: Sci Rep.;8(1):4275.

45. Wright J, Rang M. The spastic mouse and the search for an animal model of spasticity in human beings. Clin Orthop Relat Res. 1990;253:12-9.

46. Jang SH. The corticospinal tract from the viewpoint of brain rehabilitation. J Rehabil Med. 2014;46(3):193-9.
47. Martin JH. The corticospinal system: from development to motor control. Neuroscientist. 2005;11(2):161-73.

48. Graham HK, et al. Cerebral palsy. Nat Rev Dis Primers. 2016;2:15082.

49. Woolsey CN, et al. Patterns of localization in precentral and "supplementary" motor areas and their relation to the concept of a premotor area. Res Publ Assoc Res Nerv Ment Dis. 1952;30:238-64

50. Park G, Suh JH, Han SJ. Transcranial direct current stimulation for balance and gait in repetitive mild traumatic brain injury in rats. BMC Neurosci. 2021;22(1):26.

51. Novak I, et al. Early, accurate diagnosis and early intervention in cerebral palsy: advances in diagnosis and treatment. JAMA Pediatr. 2017;171(9):897-907.

52. Shevell M. Cerebral palsy to cerebral palsy spectrum disorder: time for a name change? Neurology. 2018;92:233-5.

53. Feitosa LC, et al. The effect of adapted sports in quality of life and biopsychosocial profile of children and adolescents with cerebral palSY. Rev Pau Pediatr. 2017;35(4):429-35.

54. Opheim A, et al. Walking function, pain, and fatigue in adults with cerebral palsy: a 7-year follow-up study. Dev Med Child Neurol. 2009:51(5):381-8.

55. Parkinson KN, et al. Pain in young people aged 13 to 17 years with cerebral palsy: cross-sectional, multicentre European study. Arch Dis Child. 2013;98(6):434-40

56. Ramstad K, et al. Characteristics of recurrent musculoskeletal pain in children with cerebral palsy aged 8 to 18 years. Dev Med Child Neurol. 2011;53(11):1013-8.

57. Patel DR, et al. Developmental disabilities across the lifespan. Dis Mon. 2010;56(6):304-97.

58. Liptak GS, Murphy NA. Providing a primary care medical home for children and youth with cerebral palsy. Pediatrics. 2011;128(5):e1321-9.

59. Walker AK, et al. Neuroinflammation and comorbidity of pain and depression. Pharmacol Rev. 2014;66(1):80-101.

60. Arnow BA, et al. Comorbid depression, chronic pain, and disability in primary care. Psychosom Med. 2006;68(2):262-8.

61. Chávez CE, et al. The opening of connexin 43 hemichannels alters hippocampal astrocyte function and neuronal survival in prenatally LPSexposed adult offspring. Front Cell Neurosci. 2019;13:460.

62. Tsigos C. et al. Stress: endocrine physiology and pathophysiology. In Endotext, K.R. Feingold, et al., Editors. 2000, MDText.com, Inc. Copyright @ 2000-2021, MDText.com, Inc.: South Dartmouth (MA)

63. Kantak PA, Bobrow DN, Nyby JG. Obsessive-compulsive-like behaviors in house mice are attenuated by a probiotic (Lactobacillus rhamnosus GG). Behav Pharmacol. 2014;25(1):71-9.

64. Diaz Heijtz R, et al. Normal gut microbiota modulates brain development and behavior. Proc Natl Acad Sci U S A. 2011;108(7):3047-52.

65. Pariante CM. Depression, stress and the adrenal axis. J Neuroendocrinol 2003;15(8):811-2

66. O'Mahony L, et al. Lactobacillus and bifidobacterium in irritable bowel syndrome: symptom responses and relationship to cytokine profiles. Gastroenterology. 2005;128(3):541-51.

67. Davari $\mathrm{S}$, et al. Probiotics treatment improves diabetes-induced impairment of synaptic activity and cognitive function: behavioral and electrophysiological proofs for microbiome-gut-brain axis. Neuroscience. 2013;240:287-96.

68. Bravo JA, et al. Ingestion of Lactobacillus strain regulates emotional behavior and central GABA receptor expression in a mouse via the vagus nerve. Proc Natl Acad Sci U S A. 2011;108(38):16050-5.

69. Liang S, et al. Administration of Lactobacillus helveticus NS8 improves behavioral, cognitive, and biochemical aberrations caused by chronic restraint stress. Neuroscience. 2015;310:561-77.

70. Burda JE, Bernstein AM, Sofroniew MV. Astrocyte roles in traumatic brain injury. Exp Neurol. 2016;275(Pt 3):305-15.

71. García Contreras AA, Vásquez Garibay EM, Sánchez Ramírez CA, Fafutis Morris M, Delgado Rizo V. Lactobacillus reuteri DSM 17938 and agave inulin in children with cerebral palsy and chronic constipation: a double-blind randomized placebo controlled clinical trial. Nutrients. 2020;12(10):2971. https://doi.org/10.3390/nu12102971.

72. Jacobs SE, et al. Probiotics, prematurity and neurodevelopment: followup of a randomised trial. BMJ Paediatr Open. 2017;1(1):e000176. 
73. Wang P, et al. Employing multi-omics to elucidate the hormetic response against oxidative stress exerted by $\mathrm{nC}(60)$ on Daphnia pulex. Environ Pollut. 2019;251:22-9.

74. Salles MS, Salles KS. Behavioral effect of selective and nonselective lesions of median raphe nucleus in the rat. Jpn J Physiol. 1980;30(1):105-14.

75. Yu Y, et al. Establishing a rat model of spastic cerebral palsy by targeted ethanol injection. Neural Regen Res. 2013;8(34):3255-62.

76. Andreani JC, Guma C. New animal model to mimic spastic cerebral palsy: the brain-damaged pig preparation. Neuromodulation. 2008;11(3):196-201.

77. Li B, et al. Oral bacteria colonize and compete with gut microbiota in gnotobiotic mice. Int J Oral Sci. 2019;11(1):10.

78. Wang AH, et al. Human colorectal mucosal microbiota correlates with its host niche physiology revealed by endomicroscopy. Sci Rep. 2016;6:21952

79. Zhu Y, et al. Meat, dairy and plant proteins alter bacterial composition of rat gut bacteria. Sci Rep. 2015;5:15220.

\section{Publisher's Note}

Springer Nature remains neutral with regard to jurisdictional claims in published maps and institutional affiliations.

- fast, convenient online submission

- thorough peer review by experienced researchers in your field

- rapid publication on acceptance

- support for research data, including large and complex data types

- gold Open Access which fosters wider collaboration and increased citations

- maximum visibility for your research: over 100M website views per year

At BMC, research is always in progress.

Learn more biomedcentral.com/submissions 